\title{
WEAKLY ALMOST PERIODIC FUNCTIONALS CARRIED BY HYPERCOSETS $\left({ }^{1}\right)$
}

\author{
BY \\ CHARLES F. DUNKL AND DONALD E. RAMIREZ
}

\begin{abstract}
For $G$ a compact group and $H$ a closed normal subgroup, we show that a weakly almost periodic (w.a.p.) linear functional on the Fourier algebra of $G / H$ lifts to a w.a.p. linear functional on the Fourier algebra of $G$.
\end{abstract}

In the case of a compact abelian group $G$, the dual of a closed subgroup can be identified with a quotient group of the whole dual $\hat{G}$. If $G$ is not abelian, and $H$ is a closed normal subgroup, then an identification space, $\tilde{H}$, of the dual of $H, \hat{H}$, can be identified with a hypercoset structure on $\hat{G}$. Let $H^{\perp}$ be the set of elements of $\hat{G}$ whose kernel contains $H$. (Recall $\hat{G}$ is the set of equivalence classes of continuous unitary irreducible representations of $G$.) Then $\tilde{H}$ is identified with the set of hypercosets of $H^{\perp}$, with the trivial representation $H \rightarrow\{1\}$ of course identified with $H^{\perp}$ itself. As in the abelian case, the Fourier algebra $A(G)$ of $G$ splits into a direct sum of $A(G / H)$-modules, one for each hypercoset of $H^{\perp}$. Again $A(G / H)$ itself corresponds to $H^{\perp}$. We show here that each of these modules is finitely generated, and use this result to show that weakly almost periodic (w.a.p.) linear functionals on $A(G / H)$ lift to w.a.p. linear functionals on $A(G)$ (the set of such is denoted $W(\hat{G})$ ).

We show that if $G$ has an infinite abelian homomorphic image, then the space of Fourier-Stieltjes transforms of measures on $G$ is not dense in $W(\hat{G})$, and $W(\hat{G})$ is not equal to $\mathscr{L}^{\infty}(\hat{G})$, the dual of $A(G)$. We will use some of the methods developed in our previous paper on w.a.p. functionals [6].

1. Notation and hypercosets. Let $G$ be a compact nonabelian group. Using our previous notation [3, Chapters 7,8 ] we let $\hat{G}$ denote the set of equivalence classes of continuous unitary irreducible representations of $G$. For $\alpha \in \hat{G}$, choose $T_{\alpha} \in \alpha$, then $T_{\alpha}$ is a continuous homomorphism of $G$ into $U\left(n_{\alpha}\right)$, the group of $n_{\alpha} \times n_{\alpha}$ unitary matrices where $n_{\alpha}$ is the dimension of $\alpha$. We use $T_{\alpha}(x)_{i j}$ to denote the matrix entries of $T_{\alpha}(x), 1 \leqq i, j \leqq n_{\alpha}$, and $T_{\alpha i j}$ to denote the (continuous) function $x \mapsto T_{\alpha}(x)_{i j}$. Let $V_{\alpha}=\operatorname{Sp}\left\{T_{\alpha i j}: 1 \leqq i, j \leqq n_{\alpha}\right\}$ (where Sp denotes the linear span), then $V_{\alpha}$ is an $n_{\alpha}^{2}$-dimensional space of continuous functions invariant under left

Received by the editors October 15, 1970.

AMS 1970 subject classifications. Primary 22C05, 43A40, 43-00.

Key words and phrases. Weakly almost periodic functional, quasi-uniform convergence, Fourier algebra, hypercoset, modules, Fourier-Stieltjes transforms.

$\left.{ }^{1}\right)$ This research was supported in part by NSF contract number GP-19852.

Copyright (C) 1972, American Mathematical Society 
and right translation by $G$. Further let $\chi_{\alpha}(x)=\operatorname{trace}\left(T_{\alpha}(x)\right)=\sum_{i=1}^{n} T_{\alpha}(x)_{i i}$. The function $\chi_{\alpha}$ is called the character of $\alpha$ and it, as well as $V_{\alpha}$, is independent of the choice of $T_{\alpha} \in \alpha$.

For $\alpha, \beta \in \hat{G}$ one can form the tensor product $T_{\alpha} \otimes T_{\beta}$ of the two representations. This tensor product decomposes into irreducible components: $T_{\alpha} \otimes T_{\beta}$ $\cong \sum_{\gamma \in \hat{G}} M_{\alpha \beta}(\gamma) T_{\gamma}$, where $M_{\alpha \beta}(\gamma)=\int_{G} \chi_{\alpha} \chi_{\beta} \bar{\chi}_{\gamma} d m_{G}$, a nonnegative integer $\left(m_{G}\right.$ is the normalized Haar measure on $G$ ). This decomposition can also be written in the form $\chi_{\alpha} \chi_{\beta}=\sum_{\gamma} M_{\alpha \beta}(\gamma) \chi_{\gamma}$ (a finite sum). For $E, F \subset \hat{G}$, we define

$$
E \otimes F=\left\{\gamma \in \hat{G}: M_{\alpha \beta}(\gamma) \neq 0, \text { some } \alpha \in E, \beta \in F\right\}
$$

This operation makes $\hat{G}$ into a hypergroup. For each $\alpha \in \hat{G}$, there is a conjugate $\bar{\alpha} \in \hat{G}$ such that $\chi_{\bar{\alpha}}(x)=\left(\chi_{\alpha}(x)\right)^{-}(x \in G)$. If $E \subset \hat{G}$ and $E \otimes E \subset E$, then $E$ is called a subhypergroup, and if further $\bar{E}=\{\bar{\alpha}: \alpha \in E\} \subset E$ then $E$ is called a normal subhypergroup.

For any set $S \subset G$, let $S^{\perp}=\left\{\alpha \in \hat{G}: S \subset\right.$ kernel $\left.T_{\alpha}\right\}$ then $S^{\perp}$ is a normal subhypergroup. For $E \subset \hat{G}$, let $E^{\perp}=\bigcap_{\alpha \in E}\left(\operatorname{kernel} T_{\alpha}\right)$, a closed normal subgroup of $G$. Helgason [7] has shown that if $H$ is a closed normal subgroup of $G$ then $\left(H^{\perp}\right)^{\perp}=H$.

If $E$ is a normal subhypergroup of $\hat{G}$ and $\alpha \in \hat{G}$ then $\alpha \otimes E$ is called a hypercoset of $E$. We will prove later that $\hat{G}$ is the disjoint union of hypercosets of $E$.

Let $X$ be an $n$-dimensional complex inner product space. Let $\mathscr{B}(X)$ be the space of linear maps: $X \rightarrow X$. The operator norm of $A \in \mathscr{B}(X)$ is defined to be $\|A\|_{\infty}$ $=\sup \{|A \xi|: \xi \in X,|\xi| \leqq 1\}$. The trace of $A$ is defined to be $\operatorname{Tr} A=\sum_{i=1}^{n}\left(A \xi_{i}, \xi_{i}\right)$ where $\left\{\xi_{i}\right\}_{i=1}^{n}$ is any orthonormal basis for $X$ and $(\cdot, \cdot)$ is the inner product in $X$. We define the dual norm to $\|\cdot\|_{\infty}$ by

$$
\|A\|_{1}=\sup \left\{|\operatorname{Tr}(A B)|: B \in \mathscr{B}(X),\|B\|_{\infty} \leqq 1\right\} .
$$

One can show that $\|A\|_{1}=\operatorname{Tr}(|A|)$, where $|A|=\left(A^{*} A\right)^{1 / 2}$.

Let $\phi$ be a set $\left\{\phi_{\alpha}: \alpha \in \hat{G}, \phi_{\alpha} \in \mathscr{B}\left(C^{n_{\alpha}}\right), \sup _{\alpha}\left\|\phi_{\alpha}\right\|_{\infty}<\infty\right\}$. The set of all such $\phi$ is denoted by $\mathscr{L}^{\infty}(\hat{G})$. It is a $C^{*}$-algebra under the norm $\|\phi\|_{\infty}=\sup \left\{\left\|\phi_{\alpha}\right\|_{\infty}: \alpha \in \hat{G}\right\}$ and coordinatewise operations (* denotes the operator adjoint).

Let $\mathscr{L}^{1}(\hat{G})=\left\{\phi \in \mathscr{L}^{\infty}(\hat{G}):\|\phi\|_{1}=\sum_{\alpha \in \hat{G}} n_{\alpha}\left\|\phi_{\alpha}\right\|_{1}<\infty\right\}$. Then $\mathscr{L}^{1}(\hat{G})$ with the norm $\|\cdot\|_{1}$ is a Banach space and its dual may be identified with $\mathscr{L}^{\infty}(\hat{G})$ under the pairing $\langle\phi, \psi\rangle=\sum_{\alpha} n_{\alpha} \operatorname{Tr}\left(\phi_{\alpha} \psi_{\alpha}\right) \quad\left(\phi \in \mathscr{L}^{1}(\hat{G}), \psi \in \mathscr{L}^{\infty}(\hat{G})\right)$. Let $\mu \in M(G)$, the measure algebra of $G$, then the Fourier transform of $\mu, \hat{\mu}$, is the function $\alpha \mapsto \hat{\mu}_{\alpha}$ $=\int_{G} T_{\alpha}\left(x^{-1}\right) d \mu(x) \quad(\alpha \in \hat{G})$, and $\hat{\mu} \in \mathscr{L}^{\infty}(\hat{G})$ with $\|\hat{\mu}\|_{\infty} \leqq\|\mu\|$. If $f \in C(G)$ (the continuous functions on $G)$, then $\hat{f}_{\alpha}=\int_{G} T_{\alpha}\left(x^{-1}\right) f(x) d m_{G}(x)(\alpha \in \hat{G})$.

We will now define $A(G)$, the Fourier algebra of $G$. Let

$$
A(G)=\left\{f \in C(G): \hat{f} \in \mathscr{L}^{1}(\hat{G})\right\}
$$

then $A(G)$ is in fact isomorphic to $\mathscr{L}^{1}(\hat{G})$, since for $\phi \in \mathscr{L}^{1}(\hat{G})$ the function $f(x)$ $=\sum_{\alpha \in \hat{G}} n_{\alpha} \operatorname{Tr}\left(\phi_{\alpha} T_{\alpha}(x)\right)(x \in G)$ is continuous and $\hat{f}=\phi$. Put $\|f\|_{A}=\|\hat{f}\|_{1}$. Further 
$A(G)$ is a (commutative) Banach algebra under the pointwise operations on $G$; and its dual is $\mathscr{L}^{\infty}(\hat{G})$, under the pairing

$$
\langle f, \phi\rangle=\sum_{\alpha} n_{\alpha} \operatorname{Tr}\left(\hat{f}_{\alpha} \phi_{\alpha}\right) \quad\left(f \in A(G), \phi \in \mathscr{L}^{\infty}(\hat{G})\right) ;
$$

for proofs see [3, p. 93].

Definition 1.1. For $\phi \in \mathscr{L}^{\infty}(\hat{G})$ define the carrier of $\phi$, cr $\phi=\left\{\alpha \in \hat{G}: \phi_{\alpha} \neq 0\right\}$. For $E \subset \hat{G}$, let $\mathscr{L}^{\infty}(E)=\left\{\phi \in \mathscr{L}^{\infty}(\hat{G}): \operatorname{cr} \phi \subset E\right\}$, and let $A(E)=\{f \in A(G): \operatorname{cr} \hat{f} \subset E\}$. In fact $A(E)$ is the closed span of $\left\{V_{\alpha}: \alpha \in E\right\}$.

Proposition 1.2. The spaces $A(E), E \subset \hat{G}$, are exactly the closed subspaces of $A(G)$ which are invariant under left and right translation by $G$. The dual of $A(E)$ is $\mathscr{L}^{\infty}(E)$.

Proposition 1.3. Let $E, F \subset \hat{G}$, then the closed linear span of

is equal to $A(E \otimes F)$.

$$
\{f g: f \in A(E), g \in A(F)\}
$$

Corollary 1.4. For $E \subset \hat{G}, A(E)$ is a subalgebra of $A(G)$ if and only if $E$ is a subhypergroup. Further $A(E)$ is a conjugate-closed $(f \mapsto \bar{f})$ subalgebra of $A(G)$ if and only if $E$ is a normal subhypergroup, and in that case

$$
A(E)=\left\{f \in A(G): f\left(h_{1} x h_{2}\right)=f(x) \text {, for all } h_{1}, h_{2} \in E^{\perp}, x \in G\right\},
$$

the functions in $A(G)$ constant on cosets of $E^{\perp}$, a closed normal subgroup of $G$.

COROLlaRY 1.5. If $E$ is a finite subhypergroup of $\hat{G}$ then $E$ is normal.

Proof. In fact $A(E)$ is a finite dimensional subalgebra of a conjugate closed algebra $A(G)$ and is thus itself conjugate-closed (since the maximal ideal space of $A(E)$ is a finite set).

REMARK 1.6. The Fourier algebra of a compact group $G$ is the subject of [3, Chapter 8]. Helgason [7] constructed the duality between normal subhypergroups of $\hat{G}$ and closed normal subgroups of $G$. Translation-invariant uniformly closed linear subspaces of $C(G)$ are discussed by Rider in [9]. What is observed by Iltis [8], that finite subhypergroups are normal, is implicit in Rider [9, p. 980].

2. Restrictions of representations to normal subgroups. In this section, $G$ denotes a compact group, and $H$ denotes a closed normal subgroup of $G$. Define $\hat{H}$ similarly to $\hat{G}$, and denote the character of $\gamma \in \hat{H}$ by $\xi_{\gamma}$, and let $T_{\gamma}^{H} \in \gamma$. Denote the normalized Haar measure of $H$ by $m_{H}$. There exists a natural homomorphism of $G$ into the group of automorphisms of $H$; namely, for $x \in G$, let $S_{x} h=x h x^{-1}$ $(h \in H)$, then $S_{x}$ is an automorphism of $H$. Each $S_{x}$ induces a permutation $\hat{x}$ on $\hat{H}$ such that $\xi_{\hat{x} \gamma}(h)=\xi_{\gamma}\left(S_{x} h\right)(h \in H)$. Now define an equivalence relation on $\hat{H}$ by $\gamma_{1} \sim \gamma_{2}$ if and only if $\gamma_{2}=\hat{x} \gamma_{1}$ for some $x \in G\left(\gamma_{1}, \gamma_{2} \in \hat{H}\right)$. Denote the set of such equivalence classes by $\tilde{H}$. Let $\alpha \in \hat{G}$ then $T_{\alpha} \mid H$ is a continuous unitary representation of $H$ and thus decomposes:

$$
T_{\alpha} \mid H=\sum_{\gamma \in A} a_{\gamma} T_{\gamma}^{H}
$$


where the $a_{\gamma}$ 's are nonnegative integers and only finitely many are nonzero.

THEOREM 2.1. Let $\alpha \in \hat{G}$, then there is a positive integer $N_{\alpha}$ and a class $\Gamma_{\alpha} \in \tilde{H}$ such that

$$
\chi_{\alpha} \mid H=N_{\alpha} \sum_{\gamma \in \Gamma_{\alpha}} \xi_{\gamma} .
$$

Further each equivalence class in $\tilde{H}$ is finite.

Proof. This theorem is nothing but the compact group analogue to the wellknown finite groups result (see e.g. [1, p. 278]). We sketch an argument. Write $\chi_{\alpha} \mid H=\sum_{\gamma} c_{\gamma} \xi_{\gamma}$. Now $\chi_{\alpha} \mid H$ is invariant under each $S_{x}, x \in G$, thus if $\gamma_{1} \sim \gamma_{2}$ then $c_{\gamma_{1}}=c_{\gamma_{2}}$. It remains to show that if $c_{\gamma_{1}} \neq 0$ and $c_{\gamma_{2}} \neq 0$ then $\gamma_{1} \sim \gamma_{2}$. Now $d \mu$ $=\sum_{\gamma \in \Gamma_{\alpha}} \xi_{\gamma} d m_{H}$ is a central measure in $M(G)$, and for any $\alpha \in \hat{G}, \mu$ is orthogonal to either all or none of the diagonal entry functions. If $\gamma^{\prime} \notin \Gamma_{\alpha}$, then

$$
\int_{H} \xi_{\gamma^{\prime}}\left(\sum_{\gamma \in \Gamma_{\alpha}} \xi_{\gamma}\right) d m_{H}=0
$$

Thus the class $\Gamma_{\alpha} \in \tilde{H}$ is uniquely determined and is evidently finite. However any $\gamma \in \hat{H}$ appears in the restriction of some $\alpha \in \hat{G}$ (induced representation argument) and thus any class in $\tilde{H}$ is finite.

Corollary 2.2. For any $\alpha, \beta \in \hat{G}$, either $n_{\beta} \chi_{\alpha}\left|H=n_{\alpha} \chi_{\beta}\right| H$ or $\int_{H} \chi_{\alpha} \bar{\chi}_{\beta} d m_{H}=0$.

Proof. For $\alpha, \beta \in \hat{G}$, if $\Gamma_{\alpha}=\Gamma_{\beta}$ then $n_{\alpha} / N_{\alpha}=\sum_{\gamma \in \Gamma_{\alpha}} \xi_{\gamma}(e)=n_{\beta} / N_{\beta}$ ( $e$ is identity in $G)$. If $\Gamma_{\alpha} \neq \Gamma_{\beta}$, then $\int_{H} \chi_{\alpha} \bar{\chi}_{\beta} d m_{H}=0$ by the orthogonality relations for characters of $H$.

REMARK 2.3. Rider uses this corollary in [10].

REMARK 2.4. For $\alpha \in \hat{G}, \alpha \in H^{\perp}$ if and only if $\Gamma_{\alpha}=\{\{1\}\}$ ( $\{1\}$ denotes the trivial representation $H \rightarrow\{1\})$, and in this case, $\chi_{\alpha} \mid H=n_{\alpha}=N_{\alpha}$.

TheOrem 2.5. For $\alpha, \beta \in \hat{G}, \alpha \in \beta \otimes H^{\perp}$ if and only if $\Gamma_{\alpha}=\Gamma_{\beta}$. Thus $\hat{G}$ is split into disjoint hypercosets, and these hypercosets are indexed by $\tilde{H}$.

Proof. Let $\alpha \in \beta \otimes H^{\perp}$, then there exists some $\delta \in H^{\perp}$ such that $M_{\beta \delta}(\alpha) \neq 0$, that is $\chi_{\beta} \chi_{\delta}=\chi_{\alpha}+\phi$, where $\phi$ is some nonnegative integer combination of characters. Now restrict to $H$ to obtain

$$
n_{\delta} N_{\beta} \sum_{\gamma \in \Gamma_{\beta}} \xi_{\gamma}=N_{\alpha} \sum_{\gamma \in \Gamma_{\alpha}} \xi_{\gamma}+\phi \mid H,
$$

thus $\Gamma_{\alpha} \subset \Gamma_{\beta}$, hence $\Gamma_{\alpha}=\Gamma_{\beta}$.

Conversely if $\Gamma_{\alpha}=\Gamma_{\beta}$, then $\left(\chi_{\alpha} \mid H\right)\left(\chi_{\beta} \mid H\right)^{-}=1+\phi$ (some $\phi$ as above). (This follows from the relation $M_{\gamma \bar{\gamma}}(\{1\})=\int_{H}\left|\xi_{\gamma}\right|^{2} d m_{H}=1$ for any $\gamma \in \hat{H}$.) This implies that $(\alpha \otimes \bar{\beta}) \cap H^{\perp} \neq \varnothing$. Thus there is a $\delta \in H^{\perp}$ such that $M_{\alpha \bar{\beta}}(\delta) \neq 0$, but

$$
M_{\alpha \bar{\beta}}(\delta)=\int_{G} \chi_{\alpha} \bar{\chi}_{\beta} \bar{\chi}_{\delta} d m_{G}=\int_{G} \bar{\chi}_{\alpha} \chi_{\beta} \chi_{\delta} d m_{G}=M_{\beta \delta}(\alpha)
$$

and so $\alpha \in \beta \otimes H^{\perp}$. 
Observe that the Fourier algebra of $G / H$ is isomorphic to a closed subalgebra of $A(G)$, namely $A\left(H^{\perp}\right)$ (which we will denote by $A_{H}$ ), since $(G / H)^{\wedge}$ may be identified with $H^{\perp}$. We will now decompose $A(G)$ into a direct sum of $A_{H^{-}}$-modules.

THEOREM 2.6. To each $\Gamma \in \tilde{H}$ there corresponds a closed subspace $A_{\Gamma}$ of $A(G)$ which is also an $A_{H}$-module. Further each $f \in A(G)$ has a unique decomposition $f=\sum_{\Gamma \in \tilde{H}} f_{\Gamma}$, where $f_{\Gamma} \in A_{\Gamma}$ and $\|f\|_{A}=\sum_{\Gamma \in \tilde{H}}\left\|f_{\Gamma}\right\|_{A}$. Also the $A_{\Gamma}$ 's are the minimal closed left and right translation invariant $A_{H}$-submodules of $A(G)$.

Proof. For $\Gamma \in \tilde{H}$, let $E_{\Gamma}=\left\{\alpha \in \hat{G}: \Gamma_{\alpha}=\Gamma\right\}$, that is, the hypercoset of $H$ corresponding to $\Gamma$. Then put $A_{\Gamma}=A\left(E_{\Gamma}\right)$. Clearly $\hat{G}$ is the disjoint union of $\left\{E_{\Gamma}\right\}_{\Gamma \in \tilde{H}}$, so the decomposition of $A(G)$ follows from the obvious decomposition of $\mathscr{L}^{1}(\hat{G})$. Let $\Gamma \in \tilde{H}$ and choose $\alpha \in E_{\Gamma}$ then $E_{\Gamma}=\alpha \otimes H^{\perp}$, so that $E_{\Gamma} \otimes H^{\perp}=E_{\Gamma}$ and thus, by Proposition 1.3, $A_{H} \cdot A_{\Gamma} \subset A_{\Gamma}$. So $A_{\Gamma}$ is a closed $A_{H}$-submodule of $A(G)$. If a nontrivial closed left and right translation invariant $A_{H}$-module is contained in $A_{\Gamma}$, then it is determined by some nonempty subset $F \subset E_{\Gamma}$. But if $\alpha \in F$ and $\beta \in H^{\perp}$ then $\alpha \otimes \beta \subset F$, thus $F$ is a hypercoset, hence equals $E_{\Gamma}$.

REMARK 2.7. If $G$ is abelian then each $A_{\Gamma}$ has a single generator (in the algebraic as well as the topological sense) over $A_{H}$. In the general case for $\Gamma \in \tilde{H}$ and some $\alpha \in E_{\Gamma}$, the functions $\left\{T_{\alpha i j}: 1 \leqq i, j \leqq n_{\alpha}\right\}$ generate $A_{\Gamma}$ topologically, but it is not clear that they do so algebraically. However the following is true.

THEOREM 2.8. Let $\Gamma \in \tilde{H}$, then $A_{\Gamma}$ is a finitely generated $A_{H}$-module, that is there exists $g_{1}, \ldots, g_{m} \in A_{\Gamma}$ (some $m<\infty$ ) so that each $f \in A_{\Gamma}$ may be written as $f=\sum_{i=1}^{m} k_{i} g_{i}$, with $k_{i} \in A_{H}$. Further there exists a constant $M<\infty$, such that the functions $k_{i}$ may be chosen with $\left\|k_{i}\right\|_{A} \leqq M\|f\|_{A}$.

Proof. In a paper of Dunkl [2] the following is shown: let $\tau$ be a continuous unitary representation of $H$ on a finite dimensional space $V$, and let $A(G, V)$ be the space of $V$-valued functions on $G$ with each coordinate function in $A(G)$. Define

$$
M(\tau)=\{f \in A(G, V) ; f(h x)=\tau(h) f(x) \text { for all } h \in H, x \in G\},
$$

denoted $A(\tau)$ in [2]. Then $M(\tau)$ is a finitely generated (algebraically) $A_{H}$-module.

We now point out the applicability of this theorem to the present situation. Pick $\alpha \in \Gamma$, and let $V=V_{\alpha} \mid H$. Recall $V_{\alpha}=\operatorname{Sp}\left\{T_{\alpha i j}: 1 \leqq i, j \leqq n_{\alpha}\right\}$ so that $V$ is a finite dimensional space of continuous functions on $H$, and is in fact the left and right translation invariant (by $H$ ) space generated by $\left\{\xi_{y}: \gamma \in \Gamma\right\}$. This shows that $V$ depends only on $\Gamma$, that for any $f \in A_{\Gamma}, f \mid H \in V$, and finally that $V$ is invariant under each $S_{x}, x \in G$ (that is, if $g \in V, x \in G$, then the function $h \mapsto g\left(x h x^{-1}\right)$ is in $V(h \in H))$. Observe that a continuous unitary representation $\tau$ of $H$ is realized on $V$, namely right translation, with the inner product on $V$ given by $(f, g)_{H}=\int_{H} f \bar{g} d m_{H}$ $(f, g \in V)$, and $\tau(h) f\left(h_{1}\right)=f\left(h_{1} h\right)\left(f \in V, h, h_{1} \in H\right)$.

We claim that $M(\tau)=A_{\Gamma}$, in fact if $f \in A_{\Gamma}$ then assign to each $x \in G$ the function $f(x, \cdot): h_{1} \mapsto f\left(h_{1} x\right)=(R(x) f)\left(h_{1}\right)$. Now $A_{\Gamma}$ is invariant under the right translation 
$R(x)$ so $R(x) f \mid H \in V$, thus $f(x, \cdot) \in V$. Further for $x \in G, h \in H, f\left(h x, h_{1}\right)=f\left(h_{1} h x\right)$ $=f\left(x, h_{1} h\right)=\tau(h) f\left(x, h_{1}\right)\left(h_{1} \in H\right)$, that is, $f(h x, \cdot)=\tau(h) f(x, \cdot)$. Finally to check the coordinate functions of $f(x, \cdot)$ let $g \in V$ and consider the function $x \mapsto(f(x, \cdot), g)_{H}=\int_{H} f(h x)(g(h))^{-} d m_{H}=\mu * f(x)$, where $\mu$ is the measure $\left(g\left(h^{-1}\right)\right)^{-} d m_{H}(h)$, and so $\mu * f \in A(G)$. Conversely, if $f \in M(\tau)$, so $f$ is of the form $f(x, h)$, with $f(x, \cdot) \in V$, then put $f(x)=f(x, e)$. Thus $f \in A(G)$ (by finite dimensionality of $V$, point evaluation is a bounded linear functional). Further for each $x \in G$ let $g=R(x) f \mid H$, then $g(h)=f(h x)=f(h x, e)=\tau(h) f(x, e)=f(x, h)$ so the function $g \in V$, thus $f \in A_{\Gamma}$. Hence $A_{\Gamma}=M(\tau)$ and thus there exist generators $g_{1}, \ldots, g_{m} \in A_{\Gamma}$ (some $m<\infty$ ).

Now consider the bounded linear map $T: A_{H} \times A_{H} \times \cdots \times A_{H}$ ( $m$ copies) $\rightarrow A_{\Gamma}$ defined by $T\left(k_{1}, \ldots, k_{m}\right)=\sum_{i=1}^{m} k_{i} g_{i}$. By the above paragraph $T$ is onto and so by the open mapping theorem there exists $M<\infty$ such that $\left\{T\left(k_{1}, \ldots, k_{m}\right):\left\|k_{j}\right\|_{A} \leqq M\right\}$ $\supset\left\{f \in A_{\Gamma}:\|f\|_{A} \leqq 1\right\}$.

3. Homomorphisms. Let $\pi$ be a continuous homomorphism of a compact group $G$ into a compact group $K$, and let $H$ be the kernel of $\pi$. Then $\pi$ induces the map $\pi_{1}: C(K) \rightarrow C(G)$, given by $\pi_{1} f(x)=f(\pi x), f \in C(K), x \in G$. The adjoint of $\pi_{1}$, denoted by $\pi^{*}$, takes $M(G)$ into $M(K)$. Further $\pi_{1}$ maps $A(K)$ into $A(G)$, since $A(K)$ is spanned by the continuous positive definite functions and these are preserved by $\pi_{1}$. Also $\pi_{1} \mid A(K)$ is a bounded operator on $A(K)$ since each $f \in A(K)$ is a sum $f=f_{1}-f_{2}+i\left(f_{3}-f_{4}\right), f_{i}$ positive definite and $\sum_{i=1}^{4} f_{i}(e) \leqq 2\|f\|_{A}$. Finally the adjoint of $\pi_{1} \mid A(K)$ is a bounded map $\hat{\pi}: \mathscr{L}^{\infty}(\hat{G})$ into $\mathscr{L}^{\infty}(\hat{K})$. Let $\mathscr{M}(\hat{G}), \mathscr{M}(\hat{K})$ be the closures of $M(G)^{\wedge}, M(K)^{\wedge}$ in $\mathscr{L}^{\infty}(\hat{G})$ and $\mathscr{L}^{\infty}(\hat{K})$ respectively.

Proposition 3.1. $\hat{\pi} \mathscr{M}(\hat{G}) \subset \mathscr{M}(\hat{K})$.

Proof. Let $\mu \in M(G)$, then $\hat{\mu}$ satisfies the following: $\langle f, \hat{\mu}\rangle=\int_{G} f\left(x^{-1}\right) d \mu(x)$, $f \in A(G)$. Now let $g \in A(K)$, then

$$
\langle g, \hat{\pi} \hat{\mu}\rangle=\left\langle\pi_{1} g, \hat{\mu}\right\rangle=\int_{G} g\left(\pi x^{-1}\right) d \mu(x)=\int_{K} g\left(k^{-1}\right) d \pi^{*} \mu(k)=\left\langle g,\left(\pi^{*} \mu\right)\right\rangle .
$$

Thus $\hat{\pi} \hat{\mu}=\left(\pi^{*} \mu\right)^{\wedge} \in M(K)^{\wedge}$. The continuity of $\hat{\pi}$ finishes the proof.

Observe that $\pi$ factors into $G \rightarrow G / H \rightarrow K$, where $G / H$ is identified with a closed subgroup of $K$. Further $M(G / H)$ is identified with a closed subalgebra of $M(G)$, namely $m_{H} * M(G)$ (note $m_{H}$ is an idempotent, see [3, Chapter 9]). Also $\mathscr{L}^{\infty}\left((G / H)^{\wedge}\right) \cong \mathscr{L}^{\infty}\left(H^{\llcorner}\right)$and $\mathscr{M}\left((G / H)^{\wedge}\right) \cong \mathscr{M}(\hat{G}) \cap \mathscr{L}^{\infty}\left(H^{\perp}\right)$ (since $\hat{m}_{H}$ is the projection of $\mathscr{L}^{\infty}(\hat{G})$ onto $\left.\mathscr{L}^{\infty}\left(H^{\perp}\right)\right)$.

Finally $\hat{\pi}$ takes $\mathscr{M}(\hat{G})$ onto $\mathscr{M}(\hat{K})$, or $\mathscr{L}^{\infty}(\hat{G})$ onto $\mathscr{L}^{\infty}(\hat{K})$ if and only if $\pi$ maps $G$ onto $K$, for otherwise $\pi G$ is a proper closed subgroup of $K$, and $\phi \in \hat{\pi} \mathscr{L}^{\infty}(\hat{G})$ if and only if spt $\phi \subset \pi G$ (where the support of $\phi$, spt $\phi$, is the least compact subset $E \subset K$ with the property that $f \in A(K), f=0$ on a neighborhood of $E$ implies $\langle f, \phi\rangle=0)$. 
Now we investigate the effect of $\hat{\pi}$ on $W(\hat{G})$, the weakly almost periodic (w.a.p.) elements of $\mathscr{L}^{\infty}(\hat{G})$. We state some appropriate definitions and results from our previous paper [6].

Proposition 3.2. $\mathscr{L}^{\infty}(\hat{G})$ is an $A(G)$-module. The action is defined by $\langle g, f \cdot \phi\rangle$ $=\langle f g, \phi\rangle\left(f, g \in A(G), \phi \in \mathscr{L}^{\infty}(\hat{G})\right)$, and $\|f \cdot \phi\|_{\infty} \leqq\|f\|_{A}\|\phi\|_{\infty}$. Further $\operatorname{cr}(f \cdot \phi)$ $\subset(\operatorname{cr} f)^{-} \otimes \operatorname{cr} \phi$.

Definition 3.3. For $\phi \in \mathscr{L}^{\infty}(\hat{G})$, one says that $\phi$ is weakly almost periodic if the map $f \mapsto f \cdot \phi$ is a weakly compact operator of $A(G)$ into $\mathscr{L}^{\infty}(\hat{G})(f \in A(G))$. The set of all such $\phi$ is denoted by $W(\hat{G})$.

Definition 3.4. Let $B=\left\{f \in A(G):\|f\|_{A} \leqq 1\right\}$. For $\alpha \in \hat{G}$, let $B_{\alpha}=B \cap V_{\alpha}$. Let $E=\bigcup_{\alpha \in \hat{G}} B_{\alpha}$.

Some properties of $W(\hat{G})$ (see [6]):

(1) $W(\hat{G})$ is a closed submodule of $\mathscr{L}^{\infty}(\hat{G})$.

(2) For $\phi \in \mathscr{L}^{\infty}(\hat{G})$ to be in $W(\hat{G})$ it is necessary and sufficient that $\left\{f_{n} \cdot \phi\right\}$ have a weakly convergent subsequence for any sequence $\left\{f_{n}\right\} \subset E$ (also true if $E$ is replaced by $B)$.

THEOREM 3.5. Let $\pi$ be a continuous homomorphism of $G$ into $K$ (compact groups). Then $\hat{\pi} W(\hat{G}) \subset W(\hat{K})$.

Proof. Suppose $\phi \in W(\hat{G})$, and $\left\{f_{n}\right\}$ is a bounded sequence in $A(K)$. Then $\left\{\pi_{1} f_{n}\right\}$ is a bounded sequence in $A(G)$, and there exists a subsequence such that $\left(\pi_{1} f_{n_{j}}\right) \cdot \phi$ converges weakly to $\psi \in \mathscr{L}^{\infty}(\hat{G})$. But $\hat{\pi}\left(\left(\pi_{1} f_{n_{j}}\right) \cdot \phi\right)=f_{n_{j}} \cdot(\hat{\pi} \phi)$, so $f_{n_{j}} \cdot \hat{\pi} \phi$ converges weakly to $\hat{\pi} \psi \in \mathscr{L}^{\infty}(\hat{K})$ (for $\hat{\pi}$, being strongly continuous, is weakly continuous). Hence $\hat{\pi} \phi \in W(\hat{K})$.

Henceforth we assume $\pi$ is onto $K$ so we identify $\hat{K}$ with $H^{\perp}$, and $\mathscr{L}^{\infty}(\hat{K})$ with $\mathscr{L}^{\infty}\left(H^{\perp}\right)$. We have just seen that the restriction map $\hat{\pi}: \mathscr{L}^{\infty}(\hat{G}) \rightarrow \mathscr{L}^{\infty}\left(H^{\perp}\right)$ takes $W(\hat{G})$ into $W(\hat{K})$. We will now show that in fact $\hat{\pi}\left(W(\hat{G}) \cap \mathscr{L}^{\infty}\left(H^{\perp}\right)\right)=W(\hat{K})$.

Definition 3.6. Let $\left\{\phi_{n}\right\}$ be a sequence in $\mathscr{L}^{\infty}(\hat{G})$. Say $\phi_{n} \stackrel{n}{\rightarrow} \phi \in \mathscr{L}^{\infty}(\hat{G})$ quasi-uniformly if $\left(\phi_{n}\right)_{\alpha} \stackrel{n}{\rightarrow} \phi_{\alpha}$ for each $\alpha \in \hat{G}$, and for each $\varepsilon>0, N=1,2,3, \ldots$, there exist integers $m_{1}, \ldots, m_{k} \geqq N$, such that $\min _{1 \leqq i \leqq k}\left\|\left(\phi_{m_{i}}\right)_{\alpha}-\phi_{\alpha}\right\|_{\infty}<\varepsilon$ for each $\alpha \in \hat{G}$.

THEOREM 3.7 [6]. Let $\left\{\phi_{n}\right\} \subset \mathscr{L}^{\infty}(\hat{G})$. Then $\phi_{n} \stackrel{n}{\rightarrow} \phi \in \mathscr{L}^{\infty}(\hat{G})$ weakly if and only if $\sup _{n}\left\|\phi_{n}\right\|_{\infty}<\infty$, and every subsequence of $\left\{\phi_{n}\right\}$ converges quasi-uniformly to $\phi$.

THEOREM 3.8. Let $\phi \in W(\hat{K})$, that is, $\phi \in \mathscr{L}^{\infty}\left(H^{\perp}\right)$, and for each bounded sequence, $\left\{f_{n}\right\} \subset A(K)=A_{H}$ (see previous section), $\left\{f_{n} \cdot \phi\right\}$ has a weakly convergent subsequence. Then $\phi \in W(\hat{G})$ (note $\phi_{\alpha}=0$ for $\alpha \notin H^{\perp}$ ).

Proof. Let $\left\{f_{n}\right\} \subset E=\bigcup_{\alpha} B_{\alpha}$, with $f_{n} \in B_{\alpha_{n}}, n=1,2,3, \ldots$ We must show that $\left\{f_{n} \cdot \phi\right\}$ has a weakly convergent subsequence. There are two possibilities for $\left\{\alpha_{n}\right\}$ :

(1) There are infinitely many distinct cosets $\bar{\alpha}_{n} \otimes H^{\perp}$. That is, there exists a subsequence $f_{n,}$ such that the sets $\operatorname{cr}\left(f_{n,} \cdot \phi\right) \subset \bar{\alpha}_{n,} \otimes H^{\perp}$ are all disjoint. Then $f_{n} \cdot \phi \stackrel{j}{\rightarrow} 0$ weakly by Theorem 3.7 . 
(2) Infinitely many $\alpha_{n} \in \alpha \otimes H^{\perp}$, some $\alpha \in \hat{G}$. Thus there is a bounded subsequence $f_{n_{j}}$ in $A_{\Gamma}$, where $\Gamma=\Gamma_{\alpha}$ (recall Theorem 2.6). By Theorem 2.8, there exist $g_{1}, \ldots, g_{m} \in A_{\Gamma}$ and functions $h_{i j} \in A_{H}$, and $M<\infty$, such that $f_{n_{j}}=\sum_{i=1}^{m} h_{i j} g_{j}$, and $\left\|h_{i j}\right\|_{A} \leqq M$, all $i, j$. By successively extracting subsequences from $\left\{h_{1 j}\right\},\left\{h_{2 j}\right\}$, $\ldots,\left\{h_{m j}\right\}$ and reindexing, we obtain $\psi_{1}, \ldots, \psi_{m} \in \mathscr{L}^{\infty}\left(H^{\perp}\right)$ such that $h_{i j} \cdot \phi \stackrel{j}{\rightarrow} \psi_{i}$ weakly, $i=1, \ldots, m$. The map $\psi \mapsto g_{i} \cdot \psi$ on $\mathscr{L}^{\infty}$ is strongly, hence weakly continuous, thus

$$
f_{n,} \cdot \phi=\sum_{i=1}^{m} g_{i} \cdot\left(h_{i j} \cdot \phi\right) \stackrel{j}{\rightarrow} \sum_{i=1}^{m} g_{i} \cdot \psi_{i} \quad \text { weakly }
$$

Corollary 3.9. If $W(\hat{K}) \neq \mathscr{L}^{\infty}(\hat{K})$ then $W(\hat{G}) \neq \mathscr{L}^{\infty}(\hat{G})$. If $\mathscr{M}(\hat{K}) \neq W(\hat{K})$ then $\mathscr{M}(\hat{G}) \neq W(\hat{G})$. (Recall from $[6]$ that $\mathscr{M}(\hat{G}) \subset W(\hat{G})$.)

COROLlary 3.10. If $G$ has an infinite abelian image, then $\mathscr{M}(\hat{G}) \neq W(\hat{G}) \neq \mathscr{L}^{\infty}(\hat{G})$.

Proof. If $K$ is an infinite compact abelian group, then $\mathscr{M}(\hat{K}) \neq W(\hat{K}) \neq \mathscr{L}^{\infty}(\hat{K})$ (see [3, Chapter 4] and [6]).

REMARK 3.11. In [4] we show that $W(\hat{G}) \neq \mathscr{L}^{\infty}(\hat{G})$ for any infinite compact group $G$. In [5] we show that $\mathscr{M}(\hat{G}) \neq W(\hat{G})$ for any compact group $G$ which contains an infinite abelian subgroup.

\section{BIBLIOGRAPHY}

1. C. Curtis and I. Reiner, Representation theory of finite groups and associative algebras, Pure and Appl. Math., vol. 11, Interscience, New York, 1962. MR 26 \#2519.

2. C. Dunk1, Modules over commutative Banach algebras, Monatsh. Math. 74 (1970), 6-14.

3. C. Dunkl and D. Ramirez, Topics in harmonic analysis, Appleton-Century-Crofts, New York, 1971.

4. - Existence and nonuniqueness of invariant means on $\mathscr{L}^{\infty}(\hat{G})$, Proc. Amer. Math. Soc. (to appear).

5. - Fourier-Stieltjes transforms and weakly almost periodic functionals for compact groups (to appear).

6. - Weakly almost periodic functionals on the Fourier algebra (to appear).

7. S. Helgason, Lacunary Fourier series on noncommutative groups, Proc. Amer. Math. Soc. 9 (1958), 782-790. MR 20 \#6667.

8. R. Iltis, Some algebraic structure in the dual of a compact group, Canad. J. Math. 20 (1968), 1499-1510. MR 38 \#1215.

9. D. Rider, Translation-invariant Dirichlet algebras on compact groups, Proc. Amer. Math. Soc. 17 (1966), 977-983. MR 33 \#6299.

10. - Central idempotent measures on unitary groups, Canad. J. Math. 22 (1970), 719-725.

Department of Mathematics, University of Virginia, Charlottesville, Virginia 22903 